TRANSACTIONS OF THE

AMERICAN MATHEMATICAL SOCIETY

Volume 354, Number 8 , Pages 3011-3029

S 0002-9947(02)02904-5

Article electronically published on April 3, 2002

\title{
IRREDUCIBILITY, BRILL-NOETHER LOCI, AND VOJTA'S INEQUALITY
}

\author{
THOMAS J. TUCKER AND \\ WITH AN APPENDIX BY OLIVIER DEBARRE
}

\begin{abstract}
This paper deals with generalizations of Hilbert's irreducibility theorem. The classical Hilbert irreducibility theorem states that for any cover $f$ of the projective line defined over a number field $k$, there exist infinitely many $k$-rational points on the projective line such that the fiber of $f$ over $P$ is irreducible over $k$. In this paper, we consider similar statements about algebraic points of higher degree on curves of any genus. We prove that Hilbert's irreducibility theorem admits a natural generalization to rational points on an elliptic curve and thus, via a theorem of Abramovich and Harris, to points of degree 3 or less on any curve. We also present examples that show that this generalization does not hold for points of degree 4 or more. These examples come from an earlier geometric construction of Debarre and Fahlaoui; some additional necessary facts about this construction can be found in the appendix provided by Debarre. We exhibit a connection between these irreducibility questions and the sharpness of Vojta's inequality for algebraic points on curves. In particular, we show that Vojta's inequality is not sharp for the algebraic points arising in our examples.
\end{abstract}

Given a polynomial $Q(z)$ with coefficients in $k[t]$, where $k$ is a field, one may obtain a polynomial $Q_{0}$ with coefficients in $k$ by specializing $t$ to a value $t_{0} \in k$. This specialization induces a map sending all of $k[t]$, and in particular the coefficients of $Q$, to values in $k$. If $Q$ is irreducible over $k(t)$, one might expect that many of these $Q_{0}$ will be irreducible over $k$. The classical Hilbert irreducibility theorem ([Hi], L 1, 9.1]) states that when $k$ is a number field and $Q$ is an irreducible polynomial over $k[t]$, there are infinitely many specializations $t=t_{0}$ for $t_{0} \in k$ for which the associated polynomial $Q_{0}$ is irreducible over $k$. This is equivalent to the assertion that, given any nonconstant mapping of curves $f: C^{\prime} \longrightarrow \mathbb{P}^{1}$ defined over a number field $k$, there are infinitely many points $P \in \mathbb{P}^{1}(k)$ for which the fiber $f^{-1}(P)$ is irreducible over $k$. Indeed, a rational point $P \in \mathbb{P}^{1}(k)$ gives rise to a specialization $t_{0}$ of $t$, and irreducible factors of the specialized polynomial $Q_{0}$ have a natural correspondence with points in the fiber $f^{-1}(P)$. In this paper, we explore generalizations of this theorem to points of higher degree on curves of arbitrary genus; that is to say, we replace $\mathbb{P}^{1}$ with a curve $C$ of arbitrary genus and replace the points in $\mathbb{P}^{1}(k)$ with points of bounded degree in $C(\bar{k})$. To state our results we'll need a few quick definitions.

Received by the editors May 20, 2001.

2000 Mathematics Subject Classification. Primary 11G30, 11J68.

(C)2002 American Mathematical Society 
Definition 0.1. For a curve $C$, a positive integer $d$, and a finite extension $k^{\prime}$ of $k$, we define

$$
\Gamma_{C, d}\left(k^{\prime}\right)=\left\{P \in C(\bar{k}) \mid\left[k^{\prime}(P): k^{\prime}\right] \leq d\right\} .
$$

Definition 0.2. Given a nonconstant map of curves $f: C^{\prime} \longrightarrow C$, we say that statement $H(f, d)$ holds if for some finite extension $k^{\prime}$ of $k$ there are infinitely many $P \in \Gamma_{C, d}\left(k^{\prime}\right)$ for which $f_{k^{\prime}}^{-1}(P)$ is irreducible (over $k^{\prime}$ ).

We are able to show the following.

Theorem 2.5. Let $f: C \longrightarrow E$ be a nonconstant map of degree greater than 1 from a curve $C$ to an elliptic curve $E$. Then statement $H(f, 1)$ holds.

A theorem of Abramovich and Harris $([\overline{\mathrm{A}-\mathrm{H}}]$ ) allows us to extend the theorem above to an analogous irreducibility result for points of degree 3 or less. On the other hand, there are counterexamples to such an irreducibility theorem in the case of points of degree 4 and higher. These examples are the same examples constructed by Debarre and Fahlaoui $([\mathrm{D}-\mathrm{F}])$ as counterexamples to a conjecture of Abramovich and Harris $(\underline{\mathrm{A}-\mathrm{H}}])$, though a bit more work is needed to show that they have the properties desired here.

Theorem 3.3. If $d \geq 4$, then there exists a cover of curves $f: C^{\prime} \longrightarrow C$ defined over a number field $k$, such that $\Gamma_{C, d}(k)$ is infinite but $H(f, d)$ does not hold.

The classical Hilbert irreducibility theorem can be derived from Siegel's theorem on integral points, which states that on any affine curve over a number field with more than two points at infinity, there are only finitely many integral points (for a proof along these lines, see [L 1, 9.1]). Siegel's theorem can itself be derived from Roth's theorem (for an outline of this, see [Bo, Section 2]) though historically it was proven before Roth's theorem, and was in fact derived from a weaker version of Roth's theorem. Roth's theorem states that for any algebraic number $\alpha$ and any $\epsilon>0$, there exist only finitely many rational numbers $x / y(x, y \in \mathbb{Z})$ with

$$
\left|\frac{x}{y}-\alpha\right| \leq \frac{1}{|y|^{2+\epsilon}} .
$$

The theorem of Roth is sharp, in the sense that for any real irrational algebraic number $\alpha$, there are infinitely many rational numbers $x / y(x, y \in \mathbb{Z})$ such that

$$
\left|\frac{x}{y}-\alpha\right|<\frac{1}{|y|^{2}} .
$$

The existence of such "best possible approximations to $\alpha$ " was demonstrated by Dirichlet, via a simple application of the box principle (see [Sch, Theorem 1A], for example). Given a map $f: C \longrightarrow \mathbb{P}^{1}$ and an $\alpha$ not in the ramification locus of $f$, these best possible approximations to $\alpha$ give rise to rational points $P$ on $\mathbb{P}^{1}$ for which $f^{-1}(P)$ is irreducible, as we shall see in Corollary 4.2 and Remark 4.3.

Vojta's inequality is a vast generalization of Roth's inequality. Vojta's inequality states that if $C$ is a curve over a number field $k$, then for all points $P \in C(\bar{k}) \backslash \operatorname{Supp} D$ with $[k(P): k] \leq d$, the inequality

$$
m_{S}(D, P) \leq d_{a}(P)-h_{K}(P)+\epsilon h_{A}(P)+O(1)
$$

holds, where $D$ is any effective, $k$-rational divisor without multiple components, $A$ is some ample divisor class on $C, h_{K}$ is the canonical height, and $m_{S}(D, P)$ 
is defined as the intersection $\sum_{v \in S} \frac{\left(H_{P}, H_{D}\right)_{v}}{[k(P): k]}$ of $H_{P}$ and $H_{D}$, the horizontal divisors corresponding to $P$ and $D$ on $X$. We will define all of these terms in Section 4 For the time being, let us merely note that the term $m_{S}(D, P)$ is analogous to the term $\left|\frac{x}{y}-\alpha\right|$ in Roth's theorem, that $d_{a}(P) \leq O(1)$ for rational points $P$, and that $-h_{K}(P)+\epsilon h_{A}(P)$ is approximately equal to $-\log \left(1 / y^{2+\epsilon}\right)$ when $P$ corresponds to a rational number $x / y$. As with the Dirichlet approximations, infinite families of points $P$ for which Vojta's conjecture is sharp yield infinitely many irreducible fibers $f^{-1}(P)$ when $f: C^{\prime} \longrightarrow C$ is a map of curves that doesn't ramify along $D$.

It seems natural to ask whether Vojta's inequality is sharp in the same sense that Roth's theorem is. More specifically, we might say that Vojta's inequality is sharp for points of degree $d$ or less if there exist a divisor $D$ and an infinite set $\mathcal{P}$ of points $P \in \Gamma_{C, d}(k)$ such that

$$
m_{S}(D, P)+h_{K_{C}}(P) \geq d_{a}(P)-\epsilon h(P)+O_{\epsilon}(1),
$$

for any $\epsilon>0$. In many cases, Vojta's inequality is sharp in this sense. The Dirichlet approximations described above show this for points of degree 1 on $\mathbb{P}^{1}$; other such examples are presented in [S-T 1]). It turns out, however, that having a set $\Gamma_{C, d}(k)$ of infinite cardinality does not guarantee that Vojta's inequality is sharp for points of degree $d$ or less on $C$. Indeed we show that some of the curves $C$ constructed in D-F] have the following property.

Theorem 4.7. Let $D$ be a rationally defined divisor on $C$ and choose $\epsilon>0$. Then for all $P \in \Gamma_{C, d}(k)$, the following holds:

$$
m_{S}(D, P)+h_{K_{C}}(P) \leq d_{a}(P)-(d-1-\epsilon) h(P)+O(1) .
$$

Acknowledgments. I would like to thank M. Fried, A. Granville, H. W. Lenstra, Jr., D. McKinnon, X. Song, and M. Zieve for many helpful conversations. Special thanks are due O. Debarre, who generously provided an appendix expanding upon the results of $[\mathrm{D}-\mathrm{F}]$. Finally, it is my pleasure to thank Paul Vojta, without whose help this paper would not have been possible.

\section{Definitions and Notation}

Throughout this paper, $C, C^{\prime}$, and $\tilde{C}$ will be projective, irreducible, smooth curves over a number field $k$. We will study nonconstant maps $f: C^{\prime} \longrightarrow C$. For a finite extension $k^{\prime}$ of $k$, we denote the resulting map of $k^{\prime}$-schemes as $f_{k^{\prime}}$. Given a point $P \in C(\bar{k})$, we will say that the scheme-theoretic fiber $f_{k^{\prime}}^{-1}(P)$ is irreducible if it is irreducible over $k^{\prime}$. This is the same thing as saying that $f_{k^{\prime}}^{-1}(P)$ consists of a single orbit of points in $C_{k^{\prime}}(\bar{k})$ under the Galois action of $\operatorname{Gal}\left(\bar{k}^{\prime} / k\right)$.

Recall that $C(\bar{k})$ is by definition the set of maps from Spec $\bar{k}$ to $C$. The image $Q$ of such a map is a scheme that is irreducible over $k$ and which splits into $[k(Q): k]$ points when the base is extended from $k$ to $\bar{k}$. Each element of $C(\bar{k})$ gives rise to a place of $k(C)$, in the language of function fields.

We will say that $f: C^{\prime} \longrightarrow C$ is a Galois cover if $k\left(C^{\prime}\right)$ is a Galois field extension of $k(C)$. We will say that $f$ is geometrically Galois if there exists a finite extension $k^{\prime}$ of $k$ for which $k^{\prime}\left(C^{\prime}\right)$ is Galois over $k^{\prime}(C)$.

The $d$-th symmetric product of a curve will be denoted as $C^{(d)}$. For a map $f: C^{\prime} \longrightarrow C$, the push-forward map $f_{*}$ on divisors is the map $f_{*}\left(\sum n_{i} p_{i}\right)=n_{i} f\left(P_{i}\right)$. Note that this map preserves linear equivalence (see [Fu, 1.4]).

We will use Definitions 0.2 and 0.1 from the introduction throughout this paper. 


\section{Some Irreducibility Theorems}

We begin with a simple but useful lemma.

Lemma 2.1. Suppose $f: C^{\prime} \longrightarrow C$ is a Galois cover of $C$ of degree greater than 1. Let $P$ be a point in $C(\bar{k})$ for which $f^{-1}(P)$ is reducible. Then there exists a subcover $f_{M}: C_{M}^{\prime} \longrightarrow C$ of $f$ of degree greater than 1, corresponding to a proper subgroup $M$ of $\operatorname{Gal}\left(k\left(C^{\prime}\right) / k(C)\right)$, such that $f^{-1}(P)$ contains a point $P^{\prime} \in C_{M}^{\prime}(\bar{k})$ for which $k\left(P^{\prime}\right)=k(P)$.

Proof. If $f^{-1}(P)$ is not irreducible, then every point $Q$ in it has a decomposition group $M$ that is strictly smaller than $\operatorname{Gal}\left(k\left(C^{\prime}\right) / k(C)\right)$. It follows that the image $P^{\prime}$ of $Q$ in the curve $C_{M}^{\prime}$ corresponding to the fixed field of $M$ in $k\left(C^{\prime}\right)$ has the same field of definition as $P$ (see [L 3, Proposition 7.2.4]). Of course, this means that $k\left(P^{\prime}\right)=k(P)$, as desired.

Proposition 2.2. Let $[m]: E \longrightarrow E$ be multiplication by $m$ on an elliptic curve $E$ defined over a number field $k$. Then $H([m], 1)$ holds.

Proof. We begin by observing that it will suffice to show that there is a finite extension $k^{\prime}$ of $k$ such that there is at least one nontorsion $P \in E\left(k^{\prime}\right)$ for which $[m]_{k^{\prime}}^{-1}(P)$ is irreducible. To see that this is indeed sufficient, one begins by noting that for $P \in E\left(k^{\prime}\right)$, the fiber $[m]_{k^{\prime}}^{-1}(P)$ is irreducible if and only if there exists $Q \in$ $E(\bar{k})$ such that $[m] Q=P$ and $\left[k^{\prime}(Q): k^{\prime}\right]=m^{2}$. Now, if $P \in E\left(k^{\prime}\right)$ is nontorsion and possesses such a point $Q$, then for any $r \in \mathbb{Z}^{+}$, the point $Q_{r}:=[r] P+Q$ is an $m$-division point of $[r m+1] P$ and $k^{\prime}\left(Q_{r}\right)=k^{\prime}(Q)$.

We take a map $\pi: E \longrightarrow \mathbb{P}^{1}$ and consider the composition $g=[m] \pi$. By the Hilbert irreducibility theorem ( $\mathrm{L} 1$, Chapter 9$])$ for $\mathbb{P}^{1}$, there are infinitely many $Q \in \mathbb{P}^{1}(k)$ for which $g^{-1}(Q)$ is irreducible over $k$. For such $Q$, let $P$ denote the single point of degree $\operatorname{deg} \pi$ in the fiber $\pi^{-1}(Q)$. Now, finitely many such $P$ can be torsion points. Otherwise, there would be torsion points in $E(\bar{k})$ of arbitrarily high order and fixed degree, which is impossible by the uniform boundedness theorem of [Mer]. If we extend the base to $k(P)$, it is clear by counting degrees of field extensions that $[m]_{k(P)}^{-1}(P)$ is irreducible over $k(P)$, and we are finished with our proof.

Corollary 2.3. Let $f_{i}: E_{i} \longrightarrow E$ be a finite collection of isogenies with degrees greater than 1 from elliptic curves $E_{i}$ to a fixed elliptic curve E, all of which are defined over a number field $k$. Then there exists a finite extension $k^{\prime}$ of $k$ such that there are infinitely many $P \in C\left(k^{\prime}\right)$ for which $f_{i, k^{\prime}}^{-1}(P)$ is irreducible for every $f_{i}$.

Proof. Each $f_{i}$ is dominated by a multiplication map $\left[m_{i}\right]: E \longrightarrow E$ (Mum, p. 169]). That is to say, there exist a positive integer $m_{i}$ and maps $\pi_{i}: E \longrightarrow E_{i}$ such that $\left[m_{i}\right]=f_{i} \pi_{i}$. Letting $m$ be the least common multiple of the $m_{i}$, we see that $[m]: E \longrightarrow E$ dominates all of the $f_{i}$. Clearly, if $[m]_{k^{\prime}}^{-1}(P)$ is irreducible for $P \in C\left(k^{\prime}\right)$, then $f_{i, k^{\prime}}^{-1}(P)$ is irreducible for each $f_{i}$.

Remark 2.4. It is not true that for any isogeny between elliptic curves $f: E^{\prime} \longrightarrow E$ over a number field $k$, there exist infinitely many points $P \in E(k)$ such that $f^{-1}(P)$ is irreducible over $k$. Of course, we have trivial examples when $E(k)$ is finite, but there are also examples of curves $E$ for which $E(k)$ is infinite but for which there exists an isogeny $f: E^{\prime} \longrightarrow E$ such that $f$ maps $E^{\prime}(k)$ surjectively onto $E(k)$. It 
is not hard to see, in fact, that if we have an isogeny $f: E^{\prime} \longrightarrow E$ of degree $p$, for $p$ a prime number, and if, furthermore, $E(k) \cong E^{\prime}(k) \cong \mathbb{Z}$, then, since $\hat{f} \circ f=[p]$ (where $\hat{f}$ is the dual isogeny of $f$ ), we have $\left[E^{\prime}(k): f(E(k))\right] \cdot\left[E(k): \hat{f}\left(E^{\prime}(k)\right)\right]=p$, so $\left[E^{\prime}(k): f(E(k))\right]=1$ or $\left[E(k): \hat{f}\left(E^{\prime}(k)\right)\right]=1$. Hence, either $f$ or $\hat{f}$ is surjective from rational points to rational points. Examples of such isogenies with $p$ equal to 11,5 , and 3 are given in the isogeny classes 121B, 175B, and 225A, respectively, of the table in $\mathrm{Cr}$. Thus, we see that it is necessary that we allow ourselves to enlarge $k$.

Theorem 2.5. Let $f: C \longrightarrow E$ be a nonconstant map of degree greater than 1 from a curve $C$ to an elliptic curve $E$. Then statement $H(f, 1)$ holds.

Proof. It will suffice to show that $H(g, 1)$ holds for the map $g: C^{\prime} \longrightarrow E$ from the geometric Galois closure of $C$ over $E$. We may also extend the base so that $k^{\prime}\left(C^{\prime}\right)$ is a Galois field extension of $k^{\prime}(E)$, which enables us to apply Lemma 2.1 It will suffice to show then that for some further finite extension $k^{\prime}$ there are infinitely many $P \in E\left(k^{\prime}\right)$ for which none of the fibers $g_{M, k^{\prime}}^{-1}(P)$, as $M$ runs over the proper subgroups of $\operatorname{Gal}\left(k^{\prime}\left(C^{\prime}\right) / k^{\prime}(E)\right)$, contains a point in $C_{M}^{\prime}\left(k^{\prime}\right)$. Of course, when $C_{M}^{\prime}$ is an elliptic curve $g_{M}$ is an isogeny of degree greater than 1 (since $M$ is strictly smaller than $\left.\operatorname{Gal}\left(k^{\prime}\left(C^{\prime}\right) / k^{\prime}(E)\right)\right)$. Hence, we may invoke Corollary 2.3 and conclude that there is an extension $k^{\prime}$ such that there are infinitely many $P$ with $g_{M, k^{\prime}}^{-1}(P)$ irreducible for all $M$ for which $C_{M}^{\prime}$ is elliptic. By Faltings' theorem, all $C_{M}^{\prime}$ that are not elliptic contain only finitely many rational points. Hence we are left with infinitely many $P$ for which none of the fibers $g_{M, k^{\prime}}^{-1}(P)$ contains a point in $C_{M}^{\prime}\left(k^{\prime}\right)$.

Corollary 2.6. Let $C$ be a curve and let $d \leq 3$ be a positive integer. Suppose that there exists a finite extension $k^{\prime}$ of $k$ such that $\Gamma_{C, d}\left(k^{\prime}\right)$ is infinite. Then for any nonconstant map $f: C^{\prime} \longrightarrow C$ of degree greater than 1 from a curve $C^{\prime}$ to $C$, statement $H(f, d)$ holds.

Proof. By ([A-H], Theorem 2]), when $d$ is less than or equal to $3, \Gamma_{C, d}\left(k^{\prime}\right)$ is infinite for some finite extension $k^{\prime}$ of $k$ if and only if $C$ admits a map $g: C \longrightarrow E^{\prime}$ of degree $d$ or less to a curve $E^{\prime}$ of genus 1 or less. Setting $\pi$ equal to the composition $g f$, it is easy to see that statement $H(\pi, 1)$ holds; when the genus of $E^{\prime}$ is 0 , this follows from the usual Hilbert irreducibility theorem, and when the genus of $E^{\prime}$ is 1, it follows from Theorem 2.5 It is obvious that $H(\pi, 1)$ implies $H(f, d)$.

\section{Counterexamples}

In this section we will show that for $d \geq 4$ there is a cover $f: C^{\prime} \longrightarrow C$ for which $H(f, d)$ fails to hold. The curves $C$ will be curves which admit no maps of degree $d$ or less to $\mathbb{P}^{1}$ but which have infinite $\Gamma_{C, d}(k)$.

Suppose we have a curve $C$ of genus $g \geq 2$ over a number field $k$. Let $C^{(d)}$ denote the $d$-th symmetric product of $C$ with itself. Points $P$ of degree $d$ give rise to rational points on $C^{(d)}$ by associating to $P$ the point in $C^{(d)}$ whose coordinates in $C^{d} \times_{k} \bar{k}$ are

$$
\left(P^{[1]}, \ldots, P^{[d]}\right)
$$


where $P^{[i]}$ are the conjugates of $P$ in $C(\bar{k})$ (see $[$ Frey $]$ and $[\mathrm{Si}]$ ). That this point is in fact rational follows from the fact that the action of the symmetric group $S_{d}$ on $C^{d}$ identifies all of its $\bar{k}$ conjugates; hence it is fixed by $\operatorname{Gal}(\bar{k} / k)$. Now, there is a natural map from $C^{(d)}$ to $\operatorname{Pic}^{d}(C)$. The image of $C^{(d)}$ in $\operatorname{Pic}^{d}(C)$ is denoted as $W_{d}(C)$. More generally, one denotes as $W_{d}^{r}(C)$ the set of all divisor classes $D$ (under linear equivalence) of degree $d$ such that $h^{0}(C, D) \geq r+1$ (see $\mathrm{A}-\mathrm{C}-\mathrm{G}-\mathrm{H}$. Chapter 5]). When $r=0$, the superscript zero is omitted. The varieties $W_{d}^{r}(C)$ are called Brill-Noether loci.

Since $W_{d}(C)$ is a family of divisor classes, we will write the image of a point $\left(P_{1}, \ldots, P_{d}\right)$ in $C^{(d)}(\bar{k})$ as $\left(P_{1}+\cdots+P_{d}\right)$. We may obtain an embedding $j$ of $W_{d}(C)$ into the Jacobian $J(C)=\operatorname{Pic}^{0}(C)$ of $C$ by fixing a divisor $B$ of degree $d$ on $C$ and sending $\left(P_{1}+\cdots+P_{d}\right)$ to $\left(P_{1}+\cdots+P_{d}-B\right) \in J(C)$. After extending the base, we may choose a divisor $B$ defined over $k$ so that the map $j$ will be defined over $k$.

Embedding $W_{d}(C)$ into $J(C)$ allows us to apply Faltings' theorem for subvarieties of abelian varieties ([Fa 1], [Fa 2] $)$, which tells us that $\Gamma_{C, d}\left(k^{\prime}\right)$ is infinite for some finite extension $k^{\prime}$ of $k$ if and only if $C$ admits a map of degree $d$ or less to $\mathbb{P}^{1}$ or $W_{d}(C)$ contains an abelian subvariety (see [Frey]). Faltings' theorem actually gives a bit more information. It says that if we let $Z\left(j\left(W_{d}(C)\right)\right)$ be the set of all translated abelian subvarieties of $J$ contained in $W_{d}(C)$, then $Z\left(j\left(W_{d}(C)\right)\right)$ contains all but finitely many $k^{\prime}$-valued points of $j\left(W_{d}(C)\right)$ for any finite extension $k^{\prime}$ of $k$. The set $Z\left(j\left(W_{d}(C)\right)\right)$ is called the Kawamata locus of $j\left(W_{d}(C)\right)$, and it is a closed subset of $W_{d}(C)$. ([ $\mathrm{Ka}$, Theorem 4]). Note that in general extending the base $k$ may create new $k$-rational translated abelian subvarieties of $j\left(W_{d}(C)\right)$, but it will not increase the size of the Kawamata locus of $j\left(W_{d}(C)\right)$. Since the map $j$ from $W_{d}(C)$ into $J$ is an embedding, it makes sense to speak of the Kawamata locus $Z\left(W_{d}(C)\right)$ rather than $Z\left(j\left(W_{d}(C)\right)\right)$.

Now, let $C$ be a curve that admits no maps of degree $d$ or less to curves of genus 1 or less and for which $W_{d-1}(C)$ contains no nontrivial abelian varieties. Since $C$ admits no maps of degree $d$ or less to $\mathbb{P}^{1}$, no two divisors of degree $d$ on $C$ are linear equivalent and we may identify $W_{d}(C)$ with $C^{(d)}$. Suppose that $W_{d}(C)$ contains an elliptic curve $E_{i}$ (we use the subscript $i$, because in our applications there may be more than one such elliptic curve). There is a natural map $q$ : $C \times C^{(d-1)} \longrightarrow W_{d}(C)$. This map has finite fibers, which are generically of degree $d$. Let $X_{i}$ denote the subscheme $q^{-1}\left(E_{i}\right)$. We see that the desingularization of any irreducible component of $X_{i}$ must admit a nonconstant map to $C$ by projection to the first factor of $C \times C^{(d-1)}$. It follows that the map from this desingularization to $E_{i}$ must have degree $d$, since if it had degree less than $d$, then $C$ would have to contain infinitely many points of degree less than $d$ in some extension of $k$. We see then that $X_{i}$ is irreducible and reduced. We denote its desingularization as $C_{i}$, and we denote the map from $C_{i}$ to $C \times C^{(d-1)}$ as $\psi_{i}$. We denote the projection from $C \times C^{(d-1)}$ onto the first factor as $p_{1}$. Hence, the composition $p_{1} \psi_{i}$, which we denote henceforth as $f_{i}$, gives us a map from $C_{i}$ to $C$.

Identifying $E_{i}$ with $\operatorname{Pic}^{1}\left(E_{i}\right)$, the pull-back map $(q \psi)^{*}$ sends $E_{i}$ into $W_{d}\left(C_{i}\right)$, since $q \psi_{i}$ is a map of degree $d$. We will need the following information about the image of $(q \psi)^{*}\left(E_{i}\right)$ under $f_{i *}$.

Lemma 3.1. With notation as above, $f_{i *}$ restricts to an isogeny of degree 1 on $\left(q \psi_{i}\right)^{*}\left(E_{i}\right) \subset W_{d}\left(C_{i}\right)$. 
Proof. It will suffice to show that there are infinitely many $P^{\prime} \in E_{i}(\bar{k})$ such that

$$
f_{i *}\left(q \psi_{i}\right)^{*}\left(P^{\prime}\right)=P^{\prime} .
$$

Let us choose a finite extension $k^{\prime}$ of $k$ for which $E_{i}\left(k^{\prime}\right)$ is infinite. Since $W_{d-1}(C)$ contains no nontrivial abelian varieties and $C$ admits no maps of degree $d$ or less to $\mathbb{P}^{1}$, all but finitely many of the $P^{\prime} \in E_{i}\left(k^{\prime}\right)$ correspond to points of degree $d$ over $k^{\prime}$. This allows us to write $P^{\prime}$ as $\left(P^{[1]}, \ldots, P^{[d]}\right)$ for $P^{[1]}, \ldots, P^{[d]}$ the $\bar{k}$ conjugates of a point $P$ of degree $d$ over $k^{\prime}$. Similarly, $\left(q \psi_{i}\right)_{*} P^{\prime} \in W_{d}\left(C_{i}\right)$ corresponds to a point $Q \in C_{i}(\bar{k})$ of degree $d$ over $k^{\prime}$ and can be written as $\left(Q^{[1]}, \ldots, Q^{[d]}\right)$ for $Q^{[1]}, \ldots, Q^{[d]}$ the $\bar{k}$ conjugates of a point $Q$ of degree $d$ over $k^{\prime}$. It is clear, then, that $f_{i}(Q)=P$. This implies that $f_{i *}\left(q \psi_{i}\right)^{*}\left(P^{\prime}\right)=P^{\prime}$. Since there are infinitely many such $P^{\prime}$, we are done.

Now, let $C$ be a curve which admits no maps of degree $d$ or less to curves of genus 1 or less. Suppose that $W_{d}(C)$ contains a finite collection of elliptic curves $E_{i}$, each of which is an irreducible component of the Kawamata locus of $W_{d}(C)$, and no other nontrivial abelian varieties. Suppose also that $W_{d-1}(C)$ contains no nontrivial abelian varieties. Let us extend the base $k$ for $C$ so that it contains the fields of definition of all the $E_{i}$ (this only involves taking a finite extension, since there are at most finitely many $E_{i}$ ). Since $C$ does not admit a map of degree $d$ or less to any $E_{i}$, no $C_{i}$ can be isomorphic to $C$ and the maps $f_{i}$ must all have degree greater than 1 . The $f_{i}$ induce inclusions $k(C) \subset k\left(C_{i}\right)$. Let $L$ be the Galois closure over $k(C)$ of the compositum of all the fields $k\left(C_{i}\right)$ in some algebraic closure of $k(C)$. Let us enlarge $k$ so that it contains the field of constants of $L$, let $\tilde{C}$ be the curve corresponding to the function field $L$ and let $f: \tilde{C} \longrightarrow C$ be the map from $\tilde{C}$ to $C$ coming from the inclusion of $k(C)$ in $L$.

Proposition 3.2. With $f, C$, and $\tilde{C}$ as above, statement $H(f, d)$ does not hold.

Proof. Let $k^{\prime}$ be any finite extension of $k$. All but finitely many of the points in $W_{d}(C)\left(k^{\prime}\right)$ lie in $E_{i}$ for some $i$. Now, let $P^{\prime}$ be a point in $E_{i}\left(k^{\prime}\right)$ that corresponds to a point of degree $d$ on $C$. Then $P^{\prime}$ is equal to $f_{*}\left(Q^{\prime}\right)$ for a point $Q^{\prime} \in\left(q \psi_{i}\right)^{*}\left(E_{i}\right)$. Let $Q$ be the point of degree $d$ over $k^{\prime}$ in $C_{i}(\bar{k})$ corresponding to $Q^{\prime}$. Then $f_{i}(Q)=P$, which implies that $f_{i}^{-1}(P)$ is not irreducible, since the degree of $f_{i}$ is greater than 1. Now, $f: \tilde{C} \longrightarrow C$ factors as

$$
\tilde{C} \stackrel{g_{i}}{\longrightarrow} C_{i} \stackrel{f_{i}}{\longrightarrow} C,
$$

for the natural map $g_{i}: \tilde{C} \longrightarrow C_{i}$, so this means that $f^{-1}(P)$ cannot be irreducible. We see then that all but finitely many $P \in \Gamma_{C, d}\left(k^{\prime}\right)$ have reducible fibers $f^{-1}(P)$.

We now show that for every $d \geq 4$, there exists a curve $C$ that admits no maps of degree $d$ or less to curves of genus 1 or less and such that $W_{d}(C)$ contains an elliptic curve and no abelian varieties of dimension greater than or equal 2. These curves are described in $[\overline{D-F}]$ as a family of counterexamples to a conjecture of $[\mathrm{A}-\mathrm{H}]$. We briefly summarize the construction of $[\overline{D-F}]$ as follows. For more details, see the appendix of this paper.

Let $E$ be an elliptic curve. Let $E^{(2)}$ be the symmetric product of $E$ with itself. We have a quotient map $q: E \times E \longrightarrow E^{(2)}$, a projection onto the first factor $p: E \times E \longrightarrow E$, and a summation map $s: E^{(2)} \longrightarrow E$. Let $x$ be any rational 
point on $E$ and let $(\mathbf{0})$ be the zero element in the additive structure on $E(k)$. We define the divisor $H$ on $E^{(2)}$ as $q_{*} p^{*}(\mathbf{0})$ and the divisor $F_{x}$ on $E^{(2)}$ to be $s^{*}(x)$. The linear system $\left|(d+1) H-F_{x}\right|$ contains a family of irreducible divisors. General members of this family are smooth curves $C$ ([D-F, Prop. 4.2]) that admit no maps of degree $d$ or less to curves of genus 1 or less (Prop. 5.7 and Prop. 5.14, ibid.) and for which $W_{d}(C)$ contains no abelian varieties of dimension greater than 1 (see the proof of Prop. 5.12,ibid.). Let us pick one of these $C$. It is clear that $W_{d}(C)$ contains infinitely many rational points over a suitable extension $k^{\prime}$ of $k$, since $s^{-1}(C)$ admits a map of degree $d$ to $E$ via the projection $p$. In [D-F], Debarre and Fahlaoui do not make any assertions about the Kawamata locus of $W_{d}(C)$. Debarre proves the following assertion, however, in the appendix to this paper.

Theorem A (Debarre, in appendix). If $C$ is the curve constructed above and $d \geq$ 4 , then the Kawamata locus of $W_{d}(C)$ is 1-dimensional.

The following is then an immediate consequence of the discussion above.

Theorem 3.3. If $d \geq 4$, then there exists a cover of curves $f: C^{\prime} \longrightarrow C$, defined over a number field $k$, such that $\Gamma_{C, d}(k)$ is infinite but $H(f, d)$ does not hold.

\section{Connections to Vojta's inequality}

We would like now to relate the question of Hilbert irreducibility theorems for points of higher degree to an inequality from $\mathrm{V} 2$, which we refer to as Vojta's inequality as in [S-T 1] and [S-T 2]. Vojta's inequality may be viewed as a vast generalization of Roth's theorem, one which encompasses Faltings' theorem for curves. To state Vojta's inequality precisely we will need to introduce a bit of notation. Let $C$ be a curve over a number field $k$. After taking a finite base extension of $k$, the curve $C$ has a regular model $X$ over the ring of integers $R$ of $k$ $(\underline{\mathrm{Ar}})$. At the infinite places $\sigma$ of $k$ (which correspond to embeddings $\sigma: k \hookrightarrow \mathbb{C}$ ), we may endow $C \times{ }_{\sigma} \mathbb{C}$ with an admissible Arakelov volume form (see [L 2, 4.3]). This allows us to find local and global arithmetic intersections of arithmetic divisors on $X$ as in $\mathrm{V} 2$.

An algebraic point $P \in C(k)$ gives rise to a horizontal divisor $H_{P}$ on $X$ (by taking the closure of the support of $P$ in $C$ ). We define the canonical height $h_{K}$ of a point $P \in C(k)$ to be

$$
h_{K}(P):=\frac{\left(H_{P} \cdot \omega_{X / B}\right)}{[k(P): k]},
$$

where $\omega_{X / B}$ is the canonical sheaf for $X$ over $B=\operatorname{Spec} R$, metrized with the canonical Arakelov metric (see [L 2, 4.3 and 4.5]). Note that $h_{K}$ is a Weil height for the canonical sheaf $K$ of $C$. The arithmetic discriminant $d_{a}(P)$ is then defined as

$$
d_{a}(P):=\frac{\left(H_{P} \cdot\left(\omega_{X / B}+H_{P}\right)\right)}{[k(P): k]} .
$$

This definition closely resembles the definition of the arithmetic genus of a curve on a geometric surface.

In [V 2], Vojta proves that for any curve $C$ over a number field $k$, for all points $P \in C(\bar{k}) \backslash \operatorname{Supp} D$ with $[k(P): k] \leq d$, the inequality (which we will call Vojta's inequality)

$$
m_{S}(D, P)+h_{K}(P) \leq d_{a}(P)+\epsilon h_{A}(P)+O(1)
$$


holds, where $D$ is any effective, $k$-rational divisor without multiple components, $A$ is some ample divisor class on $C, h_{K}$ is the canonical height, $S$ is a finite set of places of $K$, and $m_{S}(D, P)$ is the intersection $\sum_{v \in S} \frac{\left(H_{P}, H_{D}\right)_{v}}{[k(P): k]}$ of $H_{P}$ and $H_{D}$, the horizontal divisors corresponding to $P$ and $D$ on $X$. It is convenient to work with a fixed divisor of degree 1 rather than a general ample divisor $A$, so we set $F:=K_{C} /(2 g(C)-2)$ (unless $g(C)=1$, in which case we just fix a rationally-defined divisor of degree 1$)$. Also, set

$$
h(P):=h_{F}(P) .
$$

When $P \notin \operatorname{Supp} D$, the arithmetic intersection $\left(H_{P}, H_{D}\right)_{v}$ can be written in terms of Weil functions (see [L1, Chapter 10] for a thorough treatment of these) $\lambda_{D, v}$ as $\sum_{i=1}^{\operatorname{deg} P} \lambda_{D, v}\left(P^{[i]}\right)$, where $P^{[i]}, i=1, \ldots, \operatorname{deg} P$, are the conjugates of $P$. Hence, $m_{S}(D,-)$ will have many of the same properties as a Weil function $\lambda_{D}$. For example, if $f: C^{\prime} \longrightarrow C$ is a morphism of curves for which $f\left(P^{\prime}\right)=P$, then $m_{S}(D, P)=m_{S}\left(f^{*} D, P^{\prime}\right)$. Also, $m_{S}(D, P)$ is bounded from below for all $P$ when $D$ is effective (as is the case with Weil functions). In fact, when $D$ is effective, the contribution to $m_{S}(D, P)$ from the finite places of $S$ can never be negative, since this contribution comes from intersection numbers of effective divisors on $X$. The relationship between $m_{S}(D, P)$ and $h_{D}(P)$ is that $h_{D}(P)$ is like a sum of Weil functions for $D$ over all the places of $k$, whereas $m_{S}(D, P)$ is a sum of Weil functions over a finite set of places $S$. It is easy to see that when $D$ is effective, $h_{D}(P)-m_{S}(D, P) \geq O(1)$, a fact that we will often use.

As noted in the introduction, it is natural to ask whether or not Vojta's inequality is sharp in the same sense that Roth's theorem is sharp. We would also like to show that when one has points for which Vojta's inequality is sharp (for more on related questions see [S-T 1] and [S-T 2]), one has a very good idea of what the fibers over these points look like. We make this precise below.

Proposition 4.1. Let $C$ be a curve defined over a number field $k$ and let $D$ be a divisor of $C$ without multiple components. Suppose that $\mathcal{P}$ is a set of points of degree $d$ in $C(\bar{k}) \backslash$ Supp $D$ and that for any $\epsilon>0$ the $P$ in $\mathcal{P}$ satisfy

$$
m_{S}(D, P)+h_{K_{C}}(P) \geq d_{a}(P)-\epsilon h(P)+O_{\epsilon}(1),
$$

where $S, m_{S}, d_{a}$ and $h_{K_{C}}$ are defined as in (4.0.1) Then for any nonconstant map of curves $f: C^{\prime} \longrightarrow C$ that ramifies over at least one point not in the support of $D$, there are at most finitely many $P \in \mathcal{P}$ such that $f^{-1}(P)$ contains a point $P^{\prime} \in C^{\prime}(\bar{k})$ with $k\left(P^{\prime}\right)=k(P)$.

Proof. We begin by setting

$$
D^{\prime}=\sum_{Q \in \operatorname{Supp} f^{*} D} Q
$$

We also let $R$ denote the ramification divisor of $f$ and decompose $R$ as $R_{1}+R_{2}$, where Supp $R_{1} \subset \operatorname{Supp} D^{\prime}$, and Supp $R_{2}$ is disjoint from Supp $D^{\prime}$. We will assume that Supp $R_{2}$ is nonempty and deduce from this the desired finiteness result.

Suppose that $P^{\prime} \in C^{\prime}(\bar{k})$ has the properties that $f\left(P^{\prime}\right)=P \in \mathcal{P}$ and $k\left(P^{\prime}\right)=$ $k(P)$. Note that it is easy to see that $f^{*} D-D^{\prime}$ is equal to $R_{1}$, using the formula 
for a ramification divisor in terms of ramification indices. From this we obtain

$$
\begin{aligned}
m_{S}(D, P)-m_{S}\left(D^{\prime}, P^{\prime}\right) & \leq m_{S}\left(R_{1}, P^{\prime}\right)+O(1) \\
& \leq h_{R_{1}}\left(P^{\prime}\right)+O(1) .
\end{aligned}
$$

Since $K_{C^{\prime}}=f^{*} K_{C}+R_{1}+R_{2}$, this implies that

$$
h_{K_{C^{\prime}}}\left(P^{\prime}\right)+m_{S}\left(D^{\prime}, P^{\prime}\right) \geq h_{K_{C}}(P)+m_{S}(D, P)+h_{R_{2}}\left(P^{\prime}\right)+O(1) .
$$

Now, we choose $\epsilon>0$ in (4.1.1) small enough that

$$
h_{R_{2}}\left(P^{\prime}\right)>\epsilon\left(h(P)+h\left(P^{\prime}\right)\right)+O(1) ;
$$

this can be done because $\operatorname{deg} R_{2}$ is greater than 0 and

$$
h(P) \leq(\operatorname{deg} f) h\left(P^{\prime}\right)+O(1) .
$$

Then, since, $d_{a}(P) \geq d_{a}\left(P^{\prime}\right)$ ( $\left.\left.\mathrm{V} 1,3.4(\mathrm{e})\right]\right)$, we may combine inequalities (4.1.1]) and (4.1.2) to obtain

$$
\begin{aligned}
h_{K_{C^{\prime}}}\left(P^{\prime}\right)+m_{S}\left(D^{\prime}, P^{\prime}\right) & \geq d_{a}(P)-\epsilon h(P)+h_{R_{2}}(P)+O_{\epsilon}(1) \\
& >d_{a}\left(P^{\prime}\right)+\epsilon h\left(P^{\prime}\right)+O_{\epsilon}(1)
\end{aligned}
$$

By Vojta's inequality and Northcott's theorem, this can hold for only finitely many $P^{\prime}$.

The following corollary is a simple consequence of the above.

Corollary 4.2. Let $C, D, S$, and $\mathcal{P}$ be as in Proposition 4.1. Let $f: C^{\prime} \longrightarrow C$ be a cover of curves. Suppose that every cover $g_{M}: C_{M} \longrightarrow C$ in the Galois closure of $C^{\prime}$ over $C$ ramifies outside of $D$. Then there are only finitely many $P \in \mathcal{P}$ such that $f^{-1}(P)$ is reducible.

Proof. We note that extending the field of definition of $C$ can only reduce the degrees of the points in $\mathcal{P}$, so they continue to have bounded degree after taking a base extension. Thus, extending the base to include any extensions of the constant field that arise in forming the Galois closure of $k\left(C^{\prime}\right)$ over $k(C)$ does not change anything. Hence, we may invoke Lemma 2.1 and apply Proposition 4.1 , which proves our assertion.

Remark 4.3. When $C$ is $\mathbb{P}^{1}$ and and $S, D$, and $\mathcal{P}$ are as above, the above Corollary 4.2 implies that any map $f: C^{\prime} \longrightarrow \mathbb{P}^{1}$ that doesn't ramify along $D$ has the property that $f^{-1}(P)$ is irreducible for all but finitely many $P \in \mathcal{P}$. Thus, given a cover $f: C \longrightarrow \mathbb{P}^{1}$, we can choose a real, algebraic, irrational number $\alpha$ not in the ramification locus of $f$, set $D$ equal to the divisor given by $\alpha$, and take the Dirichlet approximations to $\alpha$ to obtain a set $\mathcal{P}$ for which Corollary 4.2 applies. Note that in the case $\operatorname{deg} D=2$, all of this can also be deduced easily from Siegel's theorem for integral points, since in this case the $\mathcal{P}$ will be integral with respect to $D$.

In light of Proposition 4.1 the curves $C$ from $[\mathrm{D}-\mathrm{F}$ discussed in the previous section would appear likely to be examples of curves with infinite $\Gamma_{C, d}(k)$ for which Vojta's inequality is not sharp for points of degree $d$ or less; the question of whether or not such curves exist was raised in [S-T 1]. We will now show that if we impose one more condition on these $C$ we will in fact obtain curves $C$ for which $\Gamma_{C, d}(k)$ is infinite but for which Vojta's inequality is not sharp for points of degree $d$ or less. This condition will ensure that at least one of the covers $f_{i}: C_{i} \longrightarrow C$ corresponding 
to an elliptic curve $E_{i}$ in $Z\left(W_{d}(C)\right)$ is not étale. This will be sufficient for our purposes, as we shall see.

It is not hard to show that since $\left|(d+1) H-F_{x}\right|$ is very ample (D-F, Prop. 4.2]), a general curve $C \in\left|(d+1) H-F_{x}\right|$ must intersect $q(\Delta)$, where $\Delta=\{(x, x) \in$ $\left.E^{2}\right\}$ and $q$ is the symmetrization map $q: E^{2} \longrightarrow E^{(2)}$, transversally. Indeed, $\left|(d+1) H-F_{x}\right|$ restricts to a very ample divisor on $q(\Delta)$, so the intersections $q(\Delta) \cap C$ are hyperplane sections. These intersections are transversal whenever the corresponding hyperplane sections are reduced, so by Bertini's theorem ( $\mathrm{Ha}$ Thm. 2.8.18]) a general element $C$ of $\left|(d+1) H-F_{x}\right|$ intersects $q(\Delta)$ transversally.

In what follows $C$ is an irreducible curve in $\left|(d+1) H-F_{x}\right|$ such that $C$ intersects $q(\Delta)$ transversally. Recall that $C$ admits no maps of degree $d$ or less to an elliptic curve or to $\mathbb{P}^{1}$, and that the Kawamata locus of $W_{d}(C)$ has dimension 1, by Theorem A of the appendix.

The inverse image of $C$ under the symmetrization map $q$ is some divisor $X$ in $E^{2}$ and, since projection induces a degree d map from $X$ onto $E$, we see that $X$ must in fact be irreducible. Hence, its desingularization $\tilde{C}$ admits a degree 2 map $f: \tilde{C} \longrightarrow C$ obtained by composing the normalization map $\tilde{C} \longrightarrow X$ with the symmetrization map $q: E^{2} \longrightarrow E^{(2)}$. We have the following lemma.

Lemma 4.4. The ramification divisor $R_{f}$ of the map defined above has degree $2(d-1)$.

Proof. We need to show that $f$ ramifies over exactly $2(d-1)$ points. We begin by showing that $C \cdot q(\Delta)=2(d-1)$. We first note that $\Delta \cdot F_{x}=4$, since $\Delta \cap F_{x}$ consists of the 2-division points of $x$. Next, we see that $\Delta \cdot H=2$, since $q^{*} H=\mathbf{0} \times C+C \times \mathbf{0}$ (as 1-cycle) and by the projection formula

$$
q_{*} \Delta \cdot H=\Delta \cdot(\mathbf{0} \times C+C \times \mathbf{0})=2 .
$$

Therefore, $C \cdot q(\Delta)=2(d+1)-4=2(d-1)$.

Now, $q$ is 2-to- 1 away from $\Delta$, so the only possible ramification points of $f$ are in $X \cap \Delta$. We see that $X$ must intersect $\Delta$ transversally because $C$ intersects $q(\Delta)$ in $2(d-1)$ distinct points, so $\Delta$ must intersect $X$ in at least $2(d-1)$ points. Since $\Delta \cdot X=2(d-1)$, this means that each intersection point has multiplicity one and is nonsingular in $X$. It follows that the normalization map $\tilde{C} \longrightarrow X$ is an isomorphism along $X \cap \Delta$ and that $f$ ramifies at exactly $2(d-1)$ points. Thus, $\operatorname{deg} R_{f}=2(d-1)$, as desired.

Now, we can give a lower bound for the arithmetic discriminant of points $P \in$ $\Gamma_{C, d}(k)$ that come from points of degree $d$ on $\tilde{C}$. We prove this lower bound below.

Lemma 4.5. Let $\epsilon>0$. All points $P \in \Gamma_{C, d}(k)$ for which there exist $P^{\prime} \in \tilde{C}(\bar{k})$ such that $f\left(P^{\prime}\right)=P$ and $k\left(P^{\prime}\right)=k(P)$ must satisfy

$$
d_{a}(P) \geq h_{K_{C}}(P)+(d-1-\epsilon) h(P)+O(1) .
$$

Proof. First, we observe that $d_{a}(P) \geq d_{a}\left(P^{\prime}\right)$, since $f\left(P^{\prime}\right)=P$ and $k\left(P^{\prime}\right)=k(P)$ ([V 1, 3.4 (e)]). Applying Vojta's inequality and using the fact that $m_{S}\left(D, P^{\prime}\right)$ is bounded from below for all $P^{\prime}$, we obtain

$$
d_{a}(P) \geq d_{a}\left(P^{\prime}\right) \geq h_{K_{\tilde{C}}}\left(P^{\prime}\right)-(\epsilon / 2) h\left(P^{\prime}\right)+O(1) .
$$


Thus, via Riemann-Hurwitz, we obtain

$$
\begin{aligned}
d_{a}(P) & \geq h_{K_{\tilde{C}}}\left(P^{\prime}\right)-(\epsilon / 2) h\left(P^{\prime}\right)+O(1) \\
& \geq h_{f^{*} K_{C}}\left(P^{\prime}\right)+h_{R_{f}}\left(P^{\prime}\right)-(\epsilon / 2) h\left(P^{\prime}\right)+O(1) \\
& \geq h_{K_{C}}(P)+h_{R_{f}}\left(P^{\prime}\right)-(\epsilon / 2) h\left(P^{\prime}\right)+O(1) .
\end{aligned}
$$

It will suffice, then, to show that

$$
h_{R_{f}}\left(P^{\prime}\right) \geq(d-1-\epsilon / 2) h(P)+O(1) .
$$

We will use the fact that the algebraic equivalence class of a divisor on a curve is determined by the degree of the divisor as well as the fact that algebraically equivalent divisors give rise to quasi-equivalent height functions (see [L 1, Prop. 4.3.3]). These facts imply that

$$
h_{R_{f}}\left(P^{\prime}\right) \geq(2(d-1)-\epsilon / 4) h\left(P^{\prime}\right)+O(1),
$$

since $h\left(P^{\prime}\right)$ is the height of $P^{\prime}$ with respect to a divisor of degree 1 . The divisor $F$, with which $h(P)=h_{F}(P)$ is defined on $C$, has degree 1 on $C$ and therefore pulls back to a divisor of degree 2 on $\tilde{C}$, so we obtain

$$
h\left(P^{\prime}\right) \geq h(P) / 2-(\epsilon /(8(d-1))) h(P)+O(1) .
$$

Combining 4.5.3) with (4.5.2), we obtain

$$
\begin{aligned}
h_{R_{f}}\left(P^{\prime}\right) & \geq((2(d-1)-\epsilon / 4)(1 / 2-\epsilon /(8(d-1))) h(P)+O(1) \\
& \geq(d-1-\epsilon / 8-\epsilon / 4) h(P)+O(1) \\
& \geq(d-1-\epsilon / 2) h(P)+O(1),
\end{aligned}
$$

as desired.

We now wish to show that the contribution of $m_{S}(D, P)$ to the left hand side of Vojta's inequality is negligible.

Lemma 4.6. Fix a rationally defined divisor $D$ on $C$ and any $\epsilon>0$. Then the inequality

$$
m_{S}(D, P) \leq \epsilon h(P)+O(1)
$$

holds for all points $P \in \Gamma_{C, d}(k) \backslash \operatorname{Supp} D$.

Proof. Recall (from the previous section) that there is a finite collection of maps $f_{i}: C_{i} \longrightarrow C$ such that for all but finitely many $P \in \Gamma_{C, d}(k)$ there exist a point $P^{\prime}$ on some $C_{i}$ and a map $\psi_{i}: C_{i} \longrightarrow E_{i}$ to an elliptic curve $E_{i}$ such that $f_{i}(P)=P^{\prime}$ and $\psi_{i}\left(P^{\prime}\right) \in E_{i}(k)$. Thus, it suffices to fix a $C_{i}$ and show that 4.6.1) holds for all $P$ for which there is a $P^{\prime} \in C_{i}$ such that $f_{i}\left(P^{\prime}\right)=P$ and $\psi_{i}\left(P^{\prime}\right) \in E_{i}(k)$. We will use the fact that Vojta's inequality takes a particularly strong from on $E_{i}$ since $K_{E_{i}}$ is trivial. We are able to pull information from $E_{i}$ back to $C_{i}$ and then push this information forward to $C$, in effect.

We begin by observing that, as noted earlier,

$$
m_{S}(D, P)=m_{S}\left(f_{i}^{*} D, P^{\prime}\right)+O(1) .
$$

As we saw in Lemma 4.5 for any $\delta>0$, we have $h\left(P^{\prime}\right)<(2+\delta) h(P)+O(1)$, so we need only show that

$$
m_{S}\left(f_{i}^{*} D, P^{\prime}\right) \leq \epsilon h\left(P^{\prime}\right)+O(1)
$$


holds for any $\epsilon>0$. Now, if we denote the divisor corresponding to $\psi_{i}\left(\operatorname{Supp} f_{i}^{*} D\right)$ as $D^{\prime}$, we see that $\left(\operatorname{deg} f_{i}\right)\left(\psi_{i}^{*} D^{\prime}\right)-f_{i}^{*} D$ is effective, because the multiplicity of any component of $f_{i}^{*} D$ is at most $\operatorname{deg} f_{i}$. Since $K_{E_{i}}$ is trivial and $d_{a}\left(\psi_{i}\left(P^{\prime}\right)\right)=0$, Vojta's inequality implies that

$$
m_{S}\left(D^{\prime}, \psi_{i}\left(P^{\prime}\right)\right) \leq \delta^{\prime} h\left(\psi_{i}\left(P^{\prime}\right)\right)+O(1)
$$

for any $\delta^{\prime}>0$. This yields

$$
\begin{aligned}
m_{S}\left(f_{i}^{*} D, P^{\prime}\right) & \leq\left(\operatorname{deg} f_{i}\right) m_{S}\left(D^{\prime}, \psi_{i}\left(P^{\prime}\right)\right)+O(1) \\
& \leq\left(\operatorname{deg} f_{i}\right) \delta^{\prime} h\left(\psi_{i}\left(P^{\prime}\right)\right)+O(1) .
\end{aligned}
$$

For any $\delta^{\prime \prime}>0$, we have

$$
h\left(\psi_{i}\left(P^{\prime}\right)\right) \leq\left(\operatorname{deg} \psi_{i}+\delta^{\prime \prime}\right) h\left(P^{\prime}\right)+O(1),
$$

so choosing $\delta^{\prime}$ and $\delta^{\prime \prime}$ such that

$$
\left(\operatorname{deg} f_{i}\right) \delta^{\prime}\left(\operatorname{deg} \psi_{i}+\delta^{\prime \prime}\right)<\epsilon
$$

gives us

$$
\begin{aligned}
m_{S}\left(f_{i}^{*} D, P^{\prime}\right) & \leq\left(\operatorname{deg} f_{i}\right) \delta^{\prime}\left(\operatorname{deg} \psi_{i}+\delta^{\prime \prime}\right) h\left(P^{\prime}\right) O(1) \\
& \leq \epsilon h\left(P^{\prime}\right)+O(1),
\end{aligned}
$$

as desired.

Finally, we are able to show that Vojta's inequality is not sharp for points of degree $d$ on $C$, although $\Gamma_{C, d}(k)$ may be infinite.

Theorem 4.7. Let $D$ be a rationally defined divisor on $C$ and choose $\epsilon>0$. Then for all $P \in \Gamma_{C, d}(k) \backslash$ Supp $D$, the following holds:

$$
m_{S}(D, P)+h_{K_{C}}(P) \leq d_{a}(P)-(d-1-\epsilon) h(P)+O(1) .
$$

Proof. By Theorem A, part a), of the appendix, $W_{d-1}(C)$ contains no nontrivial abelian varieties. Thus, $C$ has finitely many points of degree $d-1$ or less, and all but finitely many $P \in \Gamma_{C, d}(k)$ have degree equal to exactly $d$. Therefore, we may restrict to $P$ with $[k(P): k]=d$. By Lemma 4.6, this reduces to showing that

$$
d_{a}(P) \geq h_{K_{C}}(P)+(d-1+\epsilon) h(P)+O(1)
$$

for all $P \in \Gamma_{C, d}\left(k^{\prime}\right)$. We can calculate $d_{a}(P)$ by using the formula from [S-T 2] below:

$$
d_{a}(P)=h_{K}(P)+2 d h(P)-(2 / d) h_{j^{*} \Theta}\left(\left(P^{[1]}+\cdots+P^{[d]}\right)\right)+O(1),
$$

where $P^{[i]}, i=1, \ldots, d$, are the conjugates of $P$ and $\Theta$ is a certain choice of $\Theta$-divisor on $J(C)$ (different $\Theta$-divisors are algebraically equivalent, so a different choice would only effect our calculations up to an arbitrarily small $\delta>0$ ). Thus, it will suffice to show that

$$
(2 / d) h_{j^{*} \Theta}\left(\left(P^{[1]}+\cdots+P^{[d]}\right)\right) \leq(d+1+\epsilon) h(P) .
$$

As in Lemma 4.6, we will use the fact that since the Kawamata locus of $W_{d}(C)$ is 1-dimensional, there exists a finite collection of elliptic curves $E_{i} \subset W_{d}(C)$ such that $W_{d}(C)(k) \backslash \bigcup_{i} E_{i}(k)$ is finite. Let $q, X_{i}, C_{i}, \psi, p_{1}$, and $f_{i}$ be as in the previous section. It will be easier to work along a fixed $E_{i}$, as degree determines algebraic 
equivalence class along curves. We see that for $\left(P^{[1]}, \ldots, P^{[d]}\right) \in X_{i}(k)$, we have $p_{1}\left(\left(P^{[1]}, \ldots, P^{[d]}\right)\right)=P$, so that

$$
h(P)=h_{p_{1}^{*} F}\left(\left(P^{[1]}, \ldots, P^{[d]}\right)\right)+O(1),
$$

where $F$ is the divisor of degree 1 with which we define $h(P)$. Thus, if $Q \in C_{i}(\bar{k})$ is mapped to $\left(P^{[1]}, \ldots, P^{[d]}\right)$ by $\psi_{i}$, then $h(P)=h_{f_{i}^{*} F}(Q)+O(1)$. Clearly, $\operatorname{deg} f_{i}^{*} F=$ $\operatorname{deg} f_{i}$, and it is easy to see that $\operatorname{deg}\left(j q \psi_{i}\right)^{*} \Theta=d\left(j^{*} \Theta \cdot E_{i}\right)$. Hence, we have

$$
\begin{aligned}
h_{j^{*} \Theta}\left(\left(P^{[1]}+\cdots+P^{[d]}\right)\right) & =h_{\left.\left(j q \psi_{i}\right)\right)^{*} \Theta}(Q)+O(1) \\
& \leq \frac{d\left(j^{*} \Theta \cdot E_{i}\right)}{\operatorname{deg} f_{i}} h(P)+(\epsilon / 2) h(P)+O(1) .
\end{aligned}
$$

So now, using 4.7.2, we need only show that

$$
\frac{2}{d} \frac{d\left(j^{*} \Theta \cdot E_{i}\right)}{\operatorname{deg} f_{i}} h(P) \leq(d+1+\epsilon / 2) h(P)+O(1) .
$$

Noting that $\operatorname{deg} f_{i} \geq 2$, this reduces to showing that $j^{*} \Theta \cdot E_{i} \leq d+1$. Furthermore, since all the $E_{i}$ are translates of each other (as shown in the proof of [D-F] Prop. 5.14]), it will suffice to show this for a single $E_{i}$, as we have

$$
j^{*} \Theta \cdot E_{i}=\Theta \cdot j\left(E_{i}\right)=\Theta \cdot j\left(E_{\ell}\right)=j^{*} \Theta \cdot E_{\ell}
$$

for any $E_{i}$ and $E_{\ell}$. We can derive information for one of the $E_{i}$ using Lemma 4.5 One of the $E_{i}$ consists of points coming from the image of points of degree $d$ on the curve $\tilde{C}$ constructed in Lemma 4.5] under the map $f: \tilde{C} \longrightarrow C$ constructed earlier. For such points $P$, equation (4.5.1) gives a lower bound on $d_{a}(P)$ and (4.7.1) then implies that

$$
(2 / d) h_{j^{*} \Theta}\left(\left(P^{[1]}+\cdots+P^{[d]}\right)\right) \leq(d+1+\delta) h(P)+O(1)
$$

for any $\delta>0$. Working with degrees on $\tilde{C}$ (and noting that that the map $f_{i}$ in this case is just $f$, which we know has degree 2 ), we see that

$$
\frac{2}{d} \frac{d\left(j^{*} \Theta \cdot E_{i}\right)}{2} h(P) \leq(d+1+\delta) h(P)+O(1)
$$

for any $\delta>0$. Choosing $\delta$ small and letting $h(P)$ go to infinity (which we may, by Northcott's theorem, since if there are finitely many $P \in E_{i}(k)$ then the assertion of the theorem is trivial), we see that $E_{i} \cdot j^{*} \Theta \leq(d+1)$, as desired.

Remark 4.8. Since Theorem4.7 holds regardless of how large $k$ is, Vojta's inequality is not sharp for points of degree $d$ or less on $C$ in the sense of [S-T 1].

Although Vojta's inequality is not necessarily sharp, there is an easily proved upper bound on the arithmetic discriminant, namely

$$
d_{a}(P) \leq h_{K}(P)+2[k(P): k] h(P)+O(1) .
$$

In [S-T 2], this bound is used to prove the following.

Proposition 4.9 ([S-T 2, Prop. 2.4]). Let $C$ and $C^{\prime}$ be curves defined over a number field $k$, let $d$ be a positive integer, and let $f: C^{\prime} \longrightarrow C$ be a nonconstant morphism. Assume that

$$
g\left(C^{\prime}\right)-1>(d+g(C)-1) \operatorname{deg} f
$$


Then the set

$$
\left\{P \in C^{\prime}(\bar{k}) \mid[k(P): k]=d \text { and } k(f(P))=k(P)\right\}
$$

is finite.

The proof is similar to that of 4.1 Proposition 4.9 combined with Lemma 2.1 immediately yields the theorem below.

Theorem 4.10. Let $f: \tilde{C} \longrightarrow C$ be a Galois cover of curves defined over a number field $k$. Suppose that there are infinitely many $P \in \Gamma_{C, d}\left(k^{\prime}\right)$ such that $f^{-1}(P)$ is reducible. Then there exists a subcover $\phi: C^{\prime} \longrightarrow C$ of $f$ such that the ramification divisor $R_{\phi}$ satisfies

$$
\operatorname{deg} R_{\phi} \leq 2 d(\operatorname{deg} f) .
$$

Proof. By Lemma 2.1, there exists a subcover $\phi: C^{\prime} \longrightarrow C$ of $f$ such that there are infinitely many $P \in \Gamma_{C, d}\left(k^{\prime}\right)$ for which there are $P^{\prime} \in C^{\prime}(\bar{k})$ with $f\left(P^{\prime}\right)=f(P)$ and $k\left(P^{\prime}\right)=k(P)$. By Proposition 4.9, and Riemann-Hurwitz, we see that

$$
\operatorname{deg} R_{\phi}=2 g\left(C^{\prime}\right)-2-(\operatorname{deg} f)(2 g(C)-2) \leq 2 d(\operatorname{deg} f),
$$

as desired.

\section{Appendix (By Olivier Debarre)}

The aim of this appendix is to complement a construction from [D-F] (and to correct an error in the proof of Prop. 5.7 of this article). The setup is the following: let $E$ be a complex elliptic curve and let $S$ be its second symmetric product, with $s: S \rightarrow E$ the sum map. To avoid confusion between addition of divisors and addition of points on $E$, we write $(x)$ for the divisor associated with a point $x$ of $E$. We define two divisors on $S$ by setting $F=s^{-1}(\mathbf{o})$ and $H=\{(\mathbf{o})+(x) \mid x \in E\}$. For any divisor $D$ on $S$ and any $x \in E$, we set $D_{x}=D+s^{*}((x)-(\mathbf{o}))$. We denote by $\sim$ numerical equivalence for divisors.

Fix an integer $d \geq 4$ and a point $x_{0}$ on $E$, and set $L=\mathcal{O}_{S}\left((d+1) H-F_{x_{0}}\right)$. A general curve $C$ in $|L|$ is smooth of genus $\left(\begin{array}{l}d \\ 2\end{array}\right)+1$ ( $[\mathrm{D}-\mathrm{F}]$ ).

\section{On the Kawamata locus of $W_{d}(C)$}

Our aim is to prove that the Kawamata locus of $W_{d}(C)$, i.e., the union of all (translated) non-zero abelian varieties in $W_{d}(C)$, is 1-dimensional. We will analyze pencils of low degree on $C$, using as in $[\mathrm{D}-\mathrm{F}]$ the following result of Reider $([\mathrm{R}])$.

Theorem (I. Reider). Let $L$ be a nef line bundle on a smooth projective surface $S$ and let $C$ be a smooth curve in $|L|$. Let $A$ be a base-point-free $g_{\delta}^{1}$ on $C$ such that $\delta<L^{2} / 4$. There exists a divisor $D$ on $S$ such that:
a) $h^{0}(S, D) \geq 2$
b) $h^{0}(C, D-A)>0$;
c) $C \cdot D<2 \delta$;
d) $(C-D) \cdot D \leq \delta$.

For any divisor $D$ on $S$, we denote by $\bar{D}$ its restriction to $C$. 
Corollary. Let $A$ be a base-point-free $g_{\delta}^{1}$ on $C$ with $\delta \leq 2 d$ and $\delta \leq 3 d-10$. One of the following possibilities occurs:

a) there exist $x \in E$ and an effective divisor $B$ on $C$ such that $A+B \equiv 2 \bar{H}_{x}$, and $\delta \geq 2 d-4$;

b) there exist $x \in E$ and an effective divisor $B$ on $C$ such that $A+B \equiv 3 \bar{H}_{x}-\bar{F}$, and $\delta \geq 2 d-4$

c) one has $A=4 \bar{H}-2 \bar{F}$ and $\delta=2 d-2$.

Proof. A case-by-case inspection shows that the divisor $D$ in Reider's theorem satisfies one of the following: $D \sim 2 H$ and $\delta \geq 2 d-4, D \sim 3 H-F$ and $\delta \geq 2 d-4$, or $D \equiv 4 H-2 F$ and $\delta \geq 2 d-2$. In the first (resp. second) (resp. last) case, $|D|$ induces a base-point-free $g_{2 d}^{2}$ (resp. a $g_{2 d-1}^{1}$ with at most 3 distinct base points by [D-F], Prop. 4.2) (resp. a base-point-free $g_{2 d-2}^{1}$ ). Therefore, we are in case a) (resp. b)) (resp. c)).

As noted in $\left[\mathrm{D}-\mathrm{F}\right.$, the variety $W_{d}(C)$ contains a translate $E_{0}$ of $E$, to wit the image of the morphism $\psi: x \mapsto \bar{H}_{x}$.

Theorem A. Assume $C$ is general in $|L|$ and $d \geq 4$. The Kawamata locus of $W_{d}(C)$ is 1-dimensional. More precisely,

a) $W_{d-1}(C)$ contains no non-zero abelian varieties;

b) the only non-zero abelian varieties contained in $W_{d}(C)$ are translates of $E_{0}$ by torsion points;

c) for $d \geq 9$, the only non-zero abelian variety contained in $W_{d}(C)$ is $E_{0}$.

Proof. Let $A$ be a non-zero (translated) abelian variety in $W_{\delta}(C)$, with $\delta \leq d$. By a theorem of Mori (see proof of Prop. 5.4 of [D-F]), $J C / s^{*} J E$ is simple hence $A$ maps to a point in this quotient. It follows that $A$ is a translate of $E$. Take $\delta$ minimal, so that a linear system corresponding to a general point $a$ of $A$ has only one element, which we will denote by $D_{a}$, and so that the divisors $D_{a}$ have no common point. Since $E_{0}$ does not come from a morphism, one shows as in the proof of Lemma 5 of $[\mathrm{A}-\mathrm{H}]$ that $E_{0}+A \subset W_{d+\delta}^{2}(C)$.

Assume $d \geq 9$ and let $y \in E, a \in A$ and $p \in C$; the corollary of Reider's theorem applied to $\bar{H}_{y}+D_{a}-p$ yields that

a) either there exist $x \in E$ and an effective divisor $B$ on $C$ such that, for all $e \in E$, one has $\bar{H}_{y-e}+D_{a+e}-p+B \equiv 2 \bar{H}_{x}$;

b) or there exist $x \in E$ and an effective divisor $B$ on $C$ such that, for all $e \in E$, one has $\bar{H}_{y-e}+D_{a+e}-p+B \equiv 3 \bar{H}_{x}-\bar{F}$;

c) or $\delta=d-1$ and, for all $e \in E$, one has $\bar{H}_{y-e}+D_{a+e}-p \equiv 4 \bar{H}-2 \bar{F}$.

Case c) cannot occur because $p$ is fixed in $|4 \bar{H}-2 \bar{F}+p|$ by Riemann-Roch. For the same reason $B^{\prime}=B-p$ is effective in cases a) and $\mathrm{b}$ ). In case b), we get $D_{a+e}+B^{\prime} \equiv 2 \bar{H}_{x-y+e}-\bar{F}$. But this is impossible since $H^{0}\left(C, 2 \bar{H}_{x-a+e}-2 \bar{F}\right)=0$. Therefore, we are in case a), and $\bar{H}_{x-y+e}=D_{a+e}+B^{\prime}$. When $e$ varies, the lefthand side varies; the $\bar{H}_{x-y+e}$ 's having no fixed point, we must have $B^{\prime}=0$ and $\bar{H}_{x-y+e}=D_{a+e}$. It follows that $A=E_{0}$.

Assume now $d \geq 4$, and set

$$
\Gamma=\left\{\alpha \in J C \mid E_{0}+\alpha \subset W_{d}(C)\right\} .
$$

We will use Theorem 2 of $[\mathrm{A}-\mathrm{H}]$; one should however be careful: first one needs to add to the hypotheses of this theorem that the embedding $A \subset W_{d}(C)$ does not come from a morphism (this is true in our case by Prop. 5.14 of $[\mathrm{D}-\mathrm{F}]$ ). Second, the 
proof of the theorem given in $[\mathrm{A}-\mathrm{H}]$ is incomplete: the proof of Lemma 6 on which it relies is wrong when $\operatorname{dim}(A)>1$ (this fortunately does not concern us) and the case $r_{2}=3$ and $\operatorname{dim}(A)=1$ needs a separate treatment (which was provided by Abramovich in a private communication). The conclusion of the theorem is then $g(C) \leq\left(\begin{array}{l}\delta \\ 2\end{array}\right)+1$, which implies for example $\delta=d$. However, we get more from the proof, to wit that if there is equality, then $r_{k}=\left(\begin{array}{c}k+1 \\ 2\end{array}\right)-1$ for $2 \leq k \leq d$. The same reasoning shows in our case that the same holds for the sum of $k$ generic elements of $E_{0}+\alpha_{1}, \ldots, E_{0}+\alpha_{k}$, where $\alpha_{1}, \ldots, \alpha_{k} \in \Gamma$. In particular, for $a_{1}$ generic in $E_{0}+\alpha_{1}$, for $a_{2}$ generic in $E_{0}+\alpha_{2}$ and for $x$ generic in $E$, we have

$$
h^{0}\left(C,(k-2) \bar{H}_{x}+D_{a_{1}}+D_{a_{2}}\right)=\left(\begin{array}{c}
k+1 \\
2
\end{array}\right)
$$

for $2 \leq k \leq d$. Since $K_{C} \equiv(d-2) \bar{H}+\bar{H}_{-x_{0}}$, Riemann-Roch implies

$$
h^{0}\left(C, 2 \bar{H}_{x}-D_{a_{1}}\right)=1 \quad \text { and } \quad h^{0}\left(C, 3 \bar{H}_{x}-D_{a_{1}}-D_{a_{2}}\right)=1,
$$

i.e., the curves $E_{0}-\alpha_{1}$ and $E_{0}-\alpha_{1}-\alpha_{2}$ are contained in $W_{d}(C)$. This proves that $\Gamma$ is a closed (proper) subgroup of $J C$ hence is a translate of $E$ by a finite group.

\section{ERratum For}

The following proposition corrects the part of the proof of Prop. 5.7 of D-F which is incomplete (to wit the case $d=4$ ).

Proposition. Assume $d=4$ and $C$ general in $|L|$. Then $C$ has no $g_{4}^{1}$.

Proof. Let $A$ be a base-point-free $g_{\delta}^{1}$ on $C$, with $\delta \leq 4$. Following [D-F] (5.12), one constructs a rank 2 vector bundle $T$ on $S$ that fits into an exact sequence

$$
0 \rightarrow H^{0}(A)^{*} \otimes \mathcal{O}_{S} \rightarrow T \rightarrow \mathcal{O}_{C}(C-A) \rightarrow 0
$$

Note that $H^{2}(T)=0$ and $\chi(T)=10-\delta$ by Riemann-Roch. If $h=h^{0}(T)>6$, the kernel of the map $\bigwedge^{2} H^{0}(T) \rightarrow H^{0}\left(\bigwedge^{2} T\right) \simeq H^{0}(S, C) \simeq \mathbf{C}^{10}$ meets the $(2 h-3)$ dimensional set of decomposable vectors off the origin. One proceeds as in D-F (where the numbers at the top of page 246 are all wrong) to show that there exists a divisor $D$ on $S$ that fits into an exact sequence

$$
0 \rightarrow \mathcal{O}_{S}(D) \rightarrow T \rightarrow \mathcal{I}_{Z}(C-D) \rightarrow 0
$$

where $Z$ is a finite subscheme of $S$; moreover, either $D \sim 2 H$ or $D \sim 3 H-F$. Then, $h^{0}(T) \leq h^{0}(D)+h^{0}(C-D)=5$, which is a contradiction (this remark avoids the lengthy proof in $[\mathrm{D}-\mathrm{F}])$.

It follows that $h^{0}(T)=6, h^{1}(T)=0$ and $\delta=4$. Assume first that there is a non-zero morphism $u: T \rightarrow T \otimes \omega_{S}$. We argue as in $[\mathrm{L}]$ : since $H^{0}\left(\omega_{S}^{2}\right)=0$, the morphism $\bigwedge^{2} u$ vanishes hence $u$ drops rank everywhere. Then $N=(\operatorname{Im} u)^{* *}$ is a line bundle on $S$ which is a subsheaf of $T \otimes \omega_{S}$; there is a morphism $T \rightarrow N$ which is surjective off a finite subset of $S$. Note that by Riemann-Roch, one has $h^{0}\left(\mathcal{O}_{C}(C-A)\right) \geq 5>2=h^{1}\left(H^{0}(A)^{*} \otimes \mathcal{O}_{S}\right)$, hence the exact sequence $(*)$ shows that $T$ is generated by global sections off a finite subset of $S$, hence so is $N$. It follows that either $h^{0}(S, N) \geq 2$, or $N \simeq \mathcal{O}_{S}$; but the latter cannot occur since $\operatorname{Hom}\left(T, \mathcal{O}_{S}\right)=0$. Tensoring $(*)$ by $\omega_{S} \otimes N^{*}$, we see that $H^{0}\left(T \otimes \omega_{S} \otimes N^{*}\right) \neq 0$ 
implies $H^{0}\left(\omega_{C} \otimes N^{*} \otimes \mathcal{O}_{C}(-A)\right) \neq 0$ and in particular $(5 H-F) \cdot(3 H-N) \geq 4$. Furthermore, there is an exact sequence

$$
0 \rightarrow N \rightarrow T \otimes \omega_{S} \rightarrow \mathcal{I}_{Z}\left(\omega_{S}^{\otimes 2} \otimes N^{*}(C)\right) \rightarrow 0,
$$

which implies $N \cdot(H+F-N) \leq c_{2}\left(T \otimes \omega_{S}\right)=0$. A case-by-case analysis shows that the only possibility is $N \sim 2 H$; but then $H^{0}\left(\omega_{C} \otimes N^{*}(-A)\right) \neq 0$ implies $A \equiv \bar{H}_{x}$, which is not a pencil.

Hence $\operatorname{Hom}\left(T, T \otimes \omega_{S}\right)$ vanishes, and so does $H^{2}(\operatorname{End} T)$ by duality. Dualizing $(*)$ yields

$$
0 \rightarrow T^{*} \rightarrow H^{0}(A) \otimes \mathcal{O}_{S} \rightarrow \mathcal{O}_{C}(A) \rightarrow 0
$$

Tensoring by $T$, we get $H^{1}(T \otimes A)=0$. We now follow another construction of $[\mathrm{L}]$, where a moduli space $P$ is constructed which parametrizes triples $(C, A, l)$, where $C$ is a smooth curve in $|L|, A$ is a base-point-free $g_{4}^{1}$ on $C$, and $l$ is a surjective morphism $H \otimes_{\mathbf{C}} \mathcal{O}_{S} \rightarrow A$ which induces an isomorphism on global sections, two such morphisms being identified if they differ by multiplication by a non-zero scalar. Let $\pi: P \rightarrow|L|$ be the forgetful morphism. The tangent space to $P$ at $(C, A, l)$ is identified with the kernel $\tilde{H}^{0}(T \otimes A)$ of the map $H^{0}(T \otimes A) \rightarrow H^{1}(\operatorname{End} T) \stackrel{\operatorname{Tr}}{\longrightarrow}$ $H^{1}\left(\mathcal{O}_{S}\right)$; the tangent space to $|L|$ at $C$ is identified with the kernel $\tilde{H}^{0}(C, L)$ of the map $H^{0}(C, L) \rightarrow H^{1}\left(\mathcal{O}_{S}\right)$. There is an exact sequence ([L], page 304$)$

$$
\tilde{H}^{0}(T \otimes A) \stackrel{T_{(C, A, l)} \pi}{\longrightarrow} \tilde{H}^{0}(C, L) \longrightarrow(\operatorname{Ker} \mu)^{*} \longrightarrow \tilde{H}^{1}(T \otimes A)
$$

where $\mu: H^{0}(A) \otimes H^{0}\left(\omega_{C} \otimes A^{*}\right) \rightarrow H^{0}\left(\omega_{C}\right)$ is the Petri map. By the base-point-free pencil trick, its kernel is isomorphic to $H^{0}\left(\omega_{C} \otimes\left(A^{\otimes 2}\right)^{*}\right)$, which has by RiemannRoch dimension at least $h^{0}\left(A^{\otimes 2}\right)-2>0$. Since $H^{1}(T \otimes A)$ vanishes, $T_{(C, A, l)} \pi$ is not surjective, hence neither is $\pi$ by generic smoothness. This shows that there is no $g_{4}^{1}$ on a generic $C$ in $|L|$, and finishes the proof of the proposition.

\section{REFERENCES TO APPENDIX}

[AH] Abramovich, D., Harris, J., Abelian varieties and curves in $W_{d}(C)$, Comp. Math. 78 (1991), 227-238. MR 92e:14022

[DF] Debarre, O., Fahlaoui, R., Abelian varieties in $W_{d}^{r}(C)$, Comp. Math. 88 (1993), 235-249.

[L] Lazarsfeld, R., Brill-Noether-Petri without Degenerations, J. Diff. Geom. 23 (1986), 299307. MR 88b:14019

[R] Reider, I., Vector bundles of rank 2 and linear systems on algebraic surfaces, Ann. of Math. 127 (1988), 309-316. MR 89e:14038

\section{REFERENCES}

[A-H] D. Abramovich and J. Harris, Abelian varieties and curves in $W_{d}(C)$, Comp. Math. 78 (1991:2), 227-238. MR 92c:14022

[A-C-G-H] E. Arbarello, M. Cornalba, P.A. Griffiths, and J. Harris, Geometry of algebraic curves I, Springer-Verlag, New York, 1985. MR 86h:14019

[Ar] M. Artin, Lipman's proof of resolution of singularities for surfaces, in Arithmetic geometry (edited by G. Cornell and J. Silverman), Springer-Verlag, New York, 1986, pp. 267-288. MR 89b:14029

[Bo] E. Bombieri, Effective Diophantine Approximation on $\mathbb{G}_{m}$, Ann. Scuola Norm. Pisa Cl. Sci (4) 20 (1993), 61-89. MR 94m:11086

[Cr] J. E. Cremona, Algorithms for modular elliptic curves, Cambridge University Press, Cambridge, 1992 - 121B, 175B, 225A. MR 93m:11053

[D-F] O. Debarre and R. Fahlaoui, Abelian varieties in $W_{d}^{r}(C)$ and points of bounded degree on algebraic curves, Comp. Math. 88 (1993:3), 235-249. MR 94h:14028 
[Fa 1] G. Faltings, Diophantine approximation on abelian varieties, Ann. of Math. (2) 133 (1991:3), 549-576. MR 93d:11066

[Fa 2] G. Faltings, The general case of S. Lang's conjecture, in Christante, V. and Messing, W. (eds.), Barsotti symposium in algebraic geometry, Perspectives in Mathematics 15, Academic Press, San Diego, Calif., 1994 pp. 175-182. MR 95m:11061]

[Frey] G. Frey, Curves with infinitely many points of fixed degree, Israel J. Math. 85 (1994), 79-83. MR 94m:11072

[Fu $\quad$ W. Fulton, Intersection Theory, Springer-Verlag, Berlin, 1984. MR 85k:14004

[Ha] R. Hartshorne, Algebraic geometry, Springer-Verlag, Graduate Texts in Mathematics, vol. 52, New York, 1977. MR 57:3116

[Hi] D. Hilbert, Über die Irreduzibilität ganzer rationaler Funktionen mit ganzähligen Koeffzienten, J. Reine Angew. Math. 110 (1892), 104-120.

[Ka] Y. Kawamata, On Bloch's conjecture, Invent. Math. 57 (1980), 97-100. MR 81j:32030

[L 1] S. Lang, Fundamentals of diophantine geometry, Springer-Verlag, New York, 1983. MR 85j:11005

[L 2] S. Lang, Introduction to Arakelov theory, Springer-Verlag, New York, 1988. MR 89m:11059

[L 3] S. Lang, Algebra (3rd ed.), Addison-Wesley, Reading, MA, 1993. MR 86j:00003 (2nd ed.)

[Mer] L. Merel, Bornes pour la torsion des courbes elliptiques sur les corps de nombres, (French) [Bounds for the torsion of elliptic curves over number fields], Invent. Math. 124 (1996), 437-449. MR 96i:11057

[Mum] D. Mumford, Abelian varieties, Oxford University Press, London, 1970. MR 44:219

[Sch] W.M. Schmidt, Diophantine approximation, Lecture Notes in Math. 785, SpringerVerlag, New York, 1980. MR 81j:11038

[Si] J. Silverman, Rational points on symmetric products of a curve, Amer. J. Math. 113 (1991), 471-508. MR 92m:11060

[S-T 1] X. Song and T. J. Tucker, Dirichlet's Theorem, Vojta's inequality, and Vojta's conjecture, Comp. Math. 116 (1999:2), 219-238. MR 2000d:11085

[S-T 2] X. Song and T. J. Tucker, Arithmetic discriminants and morphisms of curves, Trans. AMS, 353 (2001), 1921-1936. CMP 2001:08

[V 1] P. Vojta, Arithmetic discriminants and quadratic points on curves, in Arithmetic algebraic geometry (Texel, 1989), Progr. Math. 89, Birkhäuser Boston, Boston, MA, 1991, pp. 359-376. MR 92j:11059

[V 2] P. Vojta, A generalization of theorems of Faltings and Thue-Siegel-Roth-Wirsing, J. Amer. Math. Soc. 5 (1992:4), 763-804. MR 94a:11093

Department of Mathematics, University of Georgia, Athens, Georgia 30602

E-mail address: ttucker@math.uga.edu

IRMA-Mathématique, Université Louis Pasteur, 67084 Strasbourg Cedex, France

E-mail address: debarre@math.u-strasbg.fr 